\title{
Dermatochirurgie: Tipps bei der Operation von Kindern
}

\author{
Dermatosurgery: Useful Hints for Surgery in Children
}

Autoren

Institute

\section{R. Salgo ${ }^{1}$, C. Löser ${ }^{2}$}

${ }^{1}$ Klinik für Dermatologie, Venerologie und Allergologie, J. W. Goethe Universität Frankfurt am Main (Direktor: Prof. Dr. R. Kaufmann)

${ }^{2}$ Hautklinik, Hauttumorzentrum, Klinikum der Stadt Ludwigshafen am Rhein (Direktor: Prof. Dr. E. Dippel)
Bibliografie

DOI http://dx.doi.org/

$10.1055 / \mathrm{s}-0032-1326246$

Akt Dermatol 2013; 39: 71-73

(c) Georg Thieme Verlag KG

Stuttgart · New York

ISSN 0340-2541

\section{Korrespondenzaddresse}

Dr. med. Christoph Löser

Hautklinik, Hauttumorzentrum

Klinikum Ludwigshafen

Bremserstr. 79

67063 Ludwigshafen

christoph.loeser@klilu.de

\section{Zusammenfassung \\ $\nabla$}

Dermatochirurgische Eingriffe bei Kindern fordern nicht nur manuelles Geschick und medizinische Fachkenntnisse. Tipps im Umgang mit den Beteiligten und der Berücksichtigung von Besonderheiten sollen dazu beitragen, dass der junge Patient in seiner besonderen Situation wahrgenommen wird und ein Eingriff bei korrekter Indikation zum Erfolg führt.

\section{Einleitung \\ $\nabla$}

Die Dermatologie ist ein operatives Fachgebiet. Auch Kinder und Jugendliche werden mit der Frage nach einem diagnostischen oder therapeutischen operativen Eingriff vorstellig. Hautkranke Kinder werden auch von Pädiatern betreut, die Patienten, bei denen eine Operation erforderlich ist oder scheint, dem Dermatologen schicken. Darüber hinaus sind bei speziellen Fragestellungen auch Kinderchirurgen an der operativen Therapie beteiligt.

Die Dermatochirurgie vereint den konservativen dermatologischen klinischen Blick mit der operativen Kompetenz. Jedoch sollte neben der „Liebe zur Chirurgie" und Erfahrung bei der Operation von Kindern unbedingt auch Freude im Umgang mit ihnen bestehen.

„Kinder muß man achten - und man muß sie liebhaben“ Otfried Preußler

Bei der Betreuung von operativen dermatopädiatrischen Patienten sind einige Besonderheiten zu berücksichtigen [1]. Neben dem eigentlichen Patienten gibt es auch die Eltern oder Erziehungsberechtigten (cave: nicht immer identisch). Der behandelnde Arzt findet sich in einem Spannungsfeld zwischen Kind-Eltern/Erziehungsberechtigten-Zuweiser, er muss auf die oft sehr verschiedenen Ängste und Wünsche eingehen (৫ Abb.1).

\section{Motivation und Indikationen}

Vor jeder operativen Maßnahme ist die zugrunde liegende Motivation kritisch zu hinterfragen. Handelt es sich um einen Eltern- oder Patientenwunsch oder soll der Eingriff nur auf Wunsch des Zuweisers oder nach Einschätzung des Operateurs erfolgen? Im klinischen Alltag begründet die Angst vor einer Stigmatisierung des eigenen Kindes häufig den Operationswunsch der Eltern. Hier kann versucht werden, durch entsprechende Aufklärung und ausreichend Zeit für ein Gespräch einen gemeinsam vertretbaren Weg zu finden. Erfahrungsgemäß sind Kinder im Kindergartenalter erstaunlich tolerant bzgl. „Andersartigkeit“ und vermeintlichen Makeln. Erst mit Eintritt in die Schule geht diese Toleranz offenbar verloren und eine Stigmatisierung kann tatsächlich problematisch werden. Man kann die Eltern darüber aufklären, dass der Umgang des Kindes mit seinem „Makel“ stark von dem der Eltern abhängt.

„Die größte Gefahr für Kinder geht von schlechten Eltern aus. Das ist eine statistische Wahrheit."

Hans-Ludwig Kröber

Es lässt sich ein deutlicher Unterschied im Umgang mit ihrer Dermatose erkennen zwischen Kindern, deren Eltern Sätze sagen wie „Du bist etwas ganz Besonderes, das ist ein Glücks-Fleck, den hast nur Du“, und denen, deren Eltern sich stärker als das Kind an der „Erkrankung“ stören und dies entsprechend das Kind spüren lassen bzw. auch im Umfeld so thematisieren/problematisieren.

„Man kann seine Kinder noch so gut erziehen, sie machen einem ja doch alles nach.“

Karl Kaltenegger 


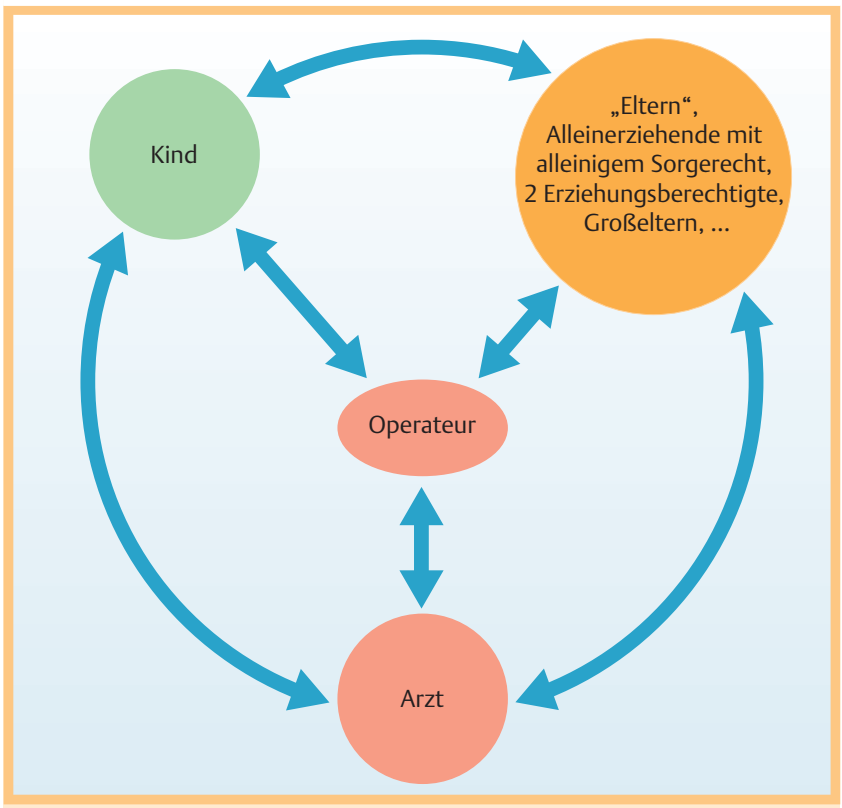

Abb. 1 Veranschaulichung von Wechselbeziehungen zwischen Operateur, Kind, Eltern, Arzt als Zuweiser und möglichen weiteren Personen: Nicht von ungefähr hat das Feld rechts oben ein besonderes Gewicht.

Nicht nur bei fachfremden Kollegen lässt sich einerseits eine häufig unbegründete Zurückhaltung bzgl. einer Probebiopsie im Kindesalter beobachten, andererseits wird gelegentlich auch eine Biopsie bei für das geschulte Auge eindeutig zuordenbarer Dermatose gefordert. Hier ist eine entsprechende Aufklärung und Rückmeldung zur Interessenwahrung des Kindes wichtig.

Hinsichtlich der Motivation des Operateurs muss kritisch hinterfragt werden, ob jeder technisch machbare Eingriff medizinisch auch sinnvoll ist.

Zusammenfassend sollte keine falsche Zurückhaltung an den Tag gelegt werden, wenn eine OP/Biopsie indiziert ist. Bei Malignitätsverdacht, Blutung, Schmerz und wenn ein Erkenntnisgewinn erwartet werden darf, dem eine therapeutische oder sonstige Konsequenz folgt, darf kein Eingriff unterbleiben, nur weil es sich um ein Kind oder einen Säugling handelt. Umgekehrt muss eine „Überdiagnostik und -therapie“ insbesondere in dieser Altersgruppe vermieden werden ( $\bullet$ Tab. 1 ).

Neben dem pädiatrisch versierten und begeisterten Dermatochirurgen sollte das ganze Team auf den Umgang mit dieser Patientengruppe eingerichtet sein und dabei helfen, eine angstfreie Atmosphäre zu schaffen. Noch stärker als dies natürlich auch bei erwachsenen Patienten gilt, darf der Behandler nicht unter Zeitdruck stehen und muss auf Verzögerungen, die durch den „Rhythmus“ des Kindes entstehen können, eingerichtet sein. „Geschrei“ gehört zum Geschäft, man darf sich dadurch nicht aus der Ruhe bringen lassen. Man sollte versuchen, die Kinder in den gesamten Ablauf möglichst einzubeziehen.

\section{„Davor"}

$\mathrm{Zu}$ beachten ist, dass alle Erziehungsberechtigten in die Maßnahme eingewilligt haben müssen. Der Eingriff sollte auch den Kindern in einer altersgerechten, nicht-ängstigenden Sprache erklärt werden (s.u.). Es sollten keine falschen Versprechungen über Schmerzfreiheit gemacht werden, sondern die zu erwartende Situation sollte kindgerecht-realistisch/ehrlich geschildert werden. Bei Anwendung eines topischen Anästhetikums muss unbedingt auf eine ausreichend lange Einwirkdauer geachtet
Tab.1 Häufige Indikationen für dermatochirurgische Eingriffe bei Kindern.

\begin{tabular}{|c|c|}
\hline Warzen & $\begin{array}{l}\text { wenn generalisiert oder in ausgewählten Problem- } \\
\text { regionen (z. B. CO }{ }_{2} \text {-Laser nach vorheriger quellender, } \\
\text { keratolytischer Behandlung; Argon-Plasma-Koagulation) }\end{array}$ \\
\hline Mollusken & Kürettage oder Zupfen mit Hilfe einer Molluskenpinzette \\
\hline $\begin{array}{l}\text { Naevus } \\
\text { flammeus }\end{array}$ & Farbstofflaser [2] \\
\hline $\begin{array}{l}\text { Kryokontakt- } \\
\text { chirurgie }\end{array}$ & $\begin{array}{l}\text { bei flachen kleinen bis mittelgroßen Hämangiomen, } \\
\text { wenn diese sich noch im Wachstum befinden (je früher, } \\
\text { je besser). Wichtig: korrekte Aufklärung, keine Entfer- } \\
\text { nung versprechen, Ziel ist die Induktion von Regression }\end{array}$ \\
\hline Naevi & $\begin{array}{l}\text { kongenital oder suspekt. Kleine Nävi: evtl. Flachschnitt, } \\
\text { Sekundärheilung. Größere Nävi: serielle Exzison (meist } \\
\text { Beginn nach 1. LJ, Abschluss zur Vermeidung von dann } \\
\text { ungünstigerer Narbenbildung vor der Pubertät) }\end{array}$ \\
\hline $\begin{array}{l}\text { Tumore/ } \\
\text { Dermatosen }\end{array}$ & $\begin{array}{l}\text { Histologische Diagnostik zum Malignitätsausschluss, } \\
\text { Diagnosesicherung vor aggressiven Therapieverfahren }\end{array}$ \\
\hline $\begin{array}{l}\text { Nagel- } \\
\text { chirurgie }\end{array}$ & $\begin{array}{l}\text { z. B. Unguis incarnatus, Abklärung von Nagelpigmentie- } \\
\text { rung bei Kaukasiern }\end{array}$ \\
\hline
\end{tabular}

werden. Bei der Lagerung können, wenn erforderlich und möglich, als „Tipp“ die Eltern mit einbezogen werden (also das Kind während des Eingriffes auf dem Arm/Schoß einer Vertrauensperson belassen).

\section{"Dabei"}

Oft stellt sich die Frage nach Anwesenheit eines Elternteils bei dem geplanten Eingriff. Grundsätzlich sind keine Zuschauer bei ärztlichen Verrichtungen vorgesehen (Musterberufsordnung §29, Abs.1, Satz2). Eine Ausnahme hiervon kann gemacht werden, wenn eine ärztliche Begründung besteht ( $\$ 29$, Abs.1, Satz4). Konkret bedeutet dies, dass der Arzt Zuschauer zulassen darf, hierzu aber nicht verpflichtet ist. Hier gilt es, im Einzelfall abzuwägen, ob ein Kind z.B. in Anwesenheit eines Elternteils schon einen Eingriff in Lokalanästhesie toleriert und hierdurch auf eine Allgemeinnarkose verzichtet werden kann oder ob umgekehrt sich die elterliche Angst eher auf das Kind überträgt und somit die Situation für alle Beteiligten von einer Abwesenheit der Eltern profitiert.

In der Regel wollen die Kinder während des Eingriffs abgelenkt werden - am besten funktioniert eine Unterhaltung mit dem Kind (zu Themen wie Schule, Haustiere, Freunde, Hobbys), in der das Gespräch dem jeweiligen „Angstlevel“ angepasst werden kann. Die Kinder können auch „Knobelspiele“ für die Hände mitbringen oder, beispielsweise bei Bauchlagerung, malen ( Abb.2). Das häufig empfohlene Musikhören kann jedoch auch Distanz schaffen und somit Ängste auslösen. Es gibt Kinder, die auch sehr versierte Ablenkungsmanöver durchschauen und lieber - natürlich in kindgerechter Sprache - detailliert über die einzelnen Maßnahmen informiert werden möchten.

Man sollte sich immer darüber im Klaren sein, dass Gespräche unter Personal gerade vor Kindern extrem ungünstig und angstauslösend sein können.

\section{Anästhesieverfahren \\ $\nabla$}

Dermatochirurgische Eingriffe bei Kindern erfolgen entweder in topischer, lokaler, allgemeiner Anästhesie oder Kombinationen dieser Verfahren. Bei kleinen Kindern muss abgewogen werden, ob der Befund ein abwartendes Verhalten rechtfertigt. Unter bei Bedarf engmaschigen klinischen Kontrollen kann erst in einem 


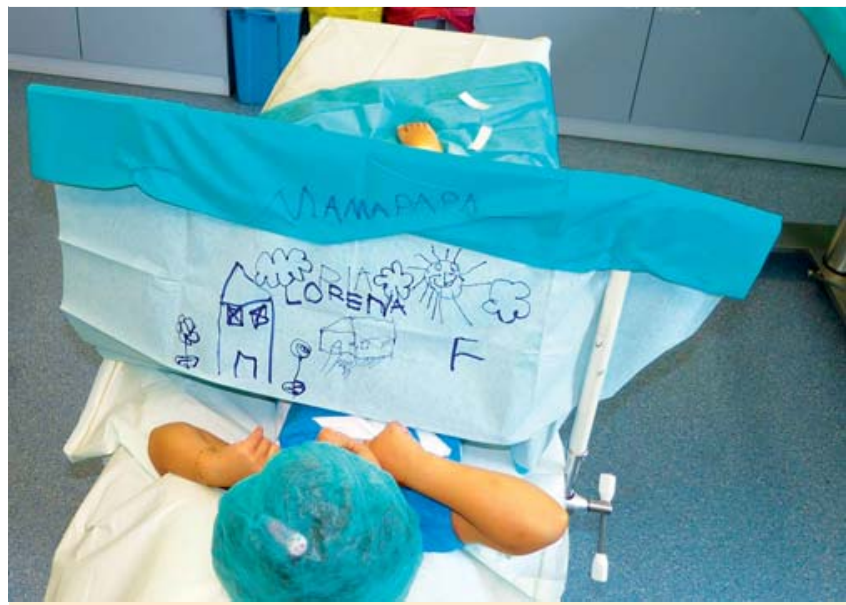

Abb.2 OP-Situation: Nutzung des Sichtschutzes als Malfläche zur Ablenkung und Unterhaltung als Beispiel für kindgerechte Kreativität im OP.

Alter, in dem ein Eingriff in Lokalanästhesie toleriert wird, operiert werden. Kinder unterscheiden sich in ihrer „Reife“, ab wann eine OP in Lokalanästhesie möglich ist. Mit der nötigen Erfahrung in der geeigneten „Herangehensweise“ ist eine Probebiospie in jedem Alter in lokaler Betäubung möglich. Bei gesundheitlichen Risiken, funktioneller Beeinträchtigung oder Deformität und evtl. auch bei zu erwartendem besseren kosmetischen Ergebnis durch einen früheren Eingriff sowie bei signifikanter psychosozialer Belastung sollte der operative Eingriff nicht hinausgezögert werden [3].

Sehr bewährt hat sich die Anwendung von topischen Lokalanästhetika (z.B. EMLA ${ }^{\circledR}$, ANESDERM $^{\circledR}$ ) (je nach Maßnahme alleine oder als betäubende Vorbehandlung). Wichtig ist, für einen ausreichenden Wirkeintritt genügend Zeit einzuplanen. Es ist günstig, die Eltern/Kinder in die korrekte Anwendung einzuweisen und die Vorbehandlung bei ambulanten Eingriffen bereits daheim durchführen zu lassen. Unbedingt muss der Höchstmengenbeschränkung Beachtung geschenkt werden, da die Gefahr einer Methämoglobinämie besteht, insbesondere für kleine Säuglinge, bei denen die Cytochrom-b5-Reduktase noch nicht in ausreichender Menge vorliegt [4].

Gründe, eine Operation in Vollnarkose durchzuführen, sind neben jungem Alter und damit fehlendem Verständnis und fehlender Kooperationsfähigkeit des Kindes die mit dem Eingriff einhergehenden, zu erwartenden Unannehmlichkeiten, die Angst und der Bedarf an Immobilisierung/Ruhigstellung/„Stillhalten“ während des Eingriffs [5]. Generell ist die Komplikationsrate bei Vollnarkosen im dermatopädiatrischen Bereich gering [6]. Bei fehlender Möglichkeit der anschließenden Überwachung in einer geeigneten stationären Einrichtung sollte mit einer Vollnarkose bis zur Vollendung des 1. Lebensjahres gewartet werden.

\section{"Danach"}

Nicht zu unterschätzen ist die Bedeutung von Belohnung und Anerkennung. Großer Beliebtheit erfreuen sich Tapferkeitsurkunden. Es kann aber auch gefragt werden, welches Motiv auf das individuell gestaltete Pflaster gemalt werden soll oder ein Stückchen Faden als „Beweis“ für die Klassenkameraden mitgegeben werden. Hier ist situations- und altersgerechter Einfallsreichtum gefordert. Die vielgeübte Praxis der Mitgabe von Sprit- zen und ähnlichem Material muss auf ein Verletzungsrisiko überprüft werden.

Die postoperativen Verhaltensregeln (Verbandswechsel, Sport-/ Schwimmverbot etc.) müssen den Eltern und den Kindern erklärt werden. Eine zusätzliche schriftliche Information sollte selbstverständlich sein.

Es muss auch daran gedacht werden, dass ein postoperativ erforderlicher Fadenzug unter Umständen problematisch sein kann. Daher sollten bereits im Vorfeld Alternativen in Erwägung gezogen werden, z.B. die Möglichkeit einer Sekundärheilung oder einer alleinigen Versorgung mit Klammerpflastern anstelle der Hautnaht (Omnistips ${ }^{\circledR}$, Steristrips ${ }^{\circledR}$ etc.).

\section{Fazit}

\section{$\nabla$}

Kinder unterscheiden sich in der dermatochirurgischen Versorgung von Erwachsenen nicht nur durch das unterschiedliche Indikationsspektrum und die Eigenschaften der kindlichen Haut, sondern insbesondere durch den stark abweichenden Anspruch an Betreuung, Umgang, Ablenkung und Einfühlungsvermögen in die kindliche Seele. Das gesamte Team sollte in der Versorgung von Kindern geschult sein. Neben dem hautkranken Kind hat der Arzt auch die Eltern als Co-Patienten wahrzunehmen. Die operative Versorgung von Kindern mag zeitaufwendiger sein als bei Erwachsenen, bringt aber auch Abwechslung und Freude in den medizinischen Alltag.

\section{Interessenkonflikt}

Die Autoren geben an, dass kein Interessenkonflikt besteht.

\section{Abstract}

\section{Dermatosurgery: Useful Hints for Surgery in Children $\nabla$}

Dermatosurgery in children calls for more than just technical skill and medical knowlegde. Hints for dealing with everybody involved and for coping with the special circumstances shall help to understand the unique situation of the young patient and lead to a successful intervention.

\section{Literatur}

1 Löser C, Möhrle M. Besonderheiten der Dermatochirurgie im Kindesalter. Hautarzt 2012; 63: $121-129$

2 Cantatore JL et al. Laser surgery: an approach to the pediatric patient. J Am Acad Dermatol 2004; 50: 165-184

3 Chen BK, Eichenfield LF. Pediatric anesthesia in dermatologic surgery: when hand-holding is not enough. Dermatol Surg 2001; 27: 10101018

4 Chen BK et al. Topical anesthetics in children: agents and techniques that equally comfort patients, parents, and clinicians. Curr Opin Pediatr 2001; 13: 324-330

5 Pittaway AJ. Pediatric dermatologic procedures performed outside the operating room. Curr Opin Anaesthesiol 2012; 25: 498-500

6 Cunningham $B B$ et al. General anesthesia for pediatric dermatologic procedures: risks and complications. Arch Dermatol 2005; 141: $573-$ 576 